\title{
Migration of Artificially Introduced Micron Size Carbon Dust in the DIII-D Divertor
}

D.L. Rudakov, W.P. West, C.P.C. Wong, N.H. Brooks, T.E. Evans, M.E. Fenstermacher, M. Groth, S.I. Krasheninnikov, C.J. Lasnier, A.G. McLean, A. Yu. Pigarov, W.M. Solomon, G.Y. Antar, J.A. Boedo, R.P. Doerner, E.M. Hollmann, A.W. Hyatt, R. Maingi, R.A. Moyer, A. Nagy, N. Nishino, L. Roquemore, P.C. Stangeby, J.G. Watkins

May 16, 2006

Journal of Nuclear Materials 
This document was prepared as an account of work sponsored by an agency of the United States Government. Neither the United States Government nor the University of California nor any of their employees, makes any warranty, express or implied, or assumes any legal liability or responsibility for the accuracy, completeness, or usefulness of any information, apparatus, product, or process disclosed, or represents that its use would not infringe privately owned rights. Reference herein to any specific commercial product, process, or service by trade name, trademark, manufacturer, or otherwise, does not necessarily constitute or imply its endorsement, recommendation, or favoring by the United States Government or the University of California. The views and opinions of authors expressed herein do not necessarily state or reflect those of the United States Government or the University of California, and shall not be used for advertising or product endorsement purposes. 


\title{
Migration of Artificially Introduced Micron Size Carbon Dust in the DIII-D Divertor
}

\author{
D.L. Rudakov ${ }^{\mathrm{a}^{*}}$, W.P. West ${ }^{\mathrm{b}}$, C.P.C. Wong ${ }^{\mathrm{b}}$, N.H. Brooks ${ }^{\mathrm{b}}$, T.E. Evans ${ }^{\mathrm{b}}$, M.E. \\ Fenstermacher $^{c}$, M. Groth ${ }^{\text {c }}$, S.I. Krasheninnikov ${ }^{\text {a }}$, C.J. Lasnier ${ }^{c}$, A.G. McLean ${ }^{\text {d }}$, A.Yu. \\ Pigarov $^{\mathrm{a}}$, W.M. Solomon ${ }^{\mathrm{e}}$, G.Y. Antar ${ }^{\mathrm{a}}$, J.A. Boedo ${ }^{\mathrm{a}}$, R.P. Doerner ${ }^{\mathrm{a}}$, E.M. Hollmann ${ }^{\mathrm{a}}$, A.W. \\ Hyatt $^{\mathrm{b}}$, R. Maingi ${ }^{\mathrm{f}}$, R.A. Moyer ${ }^{\mathrm{a}}$, A. Nagy ${ }^{\mathrm{e}}$, N. Nishino ${ }^{\mathrm{g}}$, L. Roquemore ${ }^{\mathrm{e}}$, P.C. Stangeby ${ }^{\mathrm{d}}$, J.G. \\ Watkins $^{\mathrm{h}}$ \\ ${ }^{a}$ University of California, San Diego, La Jolla, California 92093-0417, USA \\ ${ }^{b}$ General Atomics, San Diego, California 92186-5608, USA \\ ${ }^{c}$ Lawrence Livermore National Laboratory, Livermore, California 94550, USA \\ ${ }^{d}$ University of Toronto Institute for Aerospace Studies, Toronto, M3H 5T6, Canada \\ ${ }^{e}$ Princeton Plasma Physics Laboratory, Princeton, New Jersey, USA \\ ${ }^{f}$ Oak Ridge National Laboratory, Oak Ridge, Tennessee, USA \\ ${ }^{g}$ Hiroshima University, Hiroshima, Japan \\ ${ }^{h}$ Sandia National Laboratories, Albuquerque, New Mexico 87185-1129, USA
}

\begin{abstract}
Migration of pre-characterized carbon dust in a tokamak environment was studied by introducing about 30 milligrams of dust flakes $5-10 \mu \mathrm{m}$ in diameter in the lower divertor of DIII-D using the DiMES sample holder. The dust was exposed to high power ELMing Hmode discharges in lower-single-null magnetic configuration with the strike points swept across the divertor floor. When the outer strike point (OSP) passed over the dust holder exposing it to high particle and heat fluxes, part of the dust was injected into the plasma. In about $0.1 \mathrm{sec}$ following the OSP pass over the dust, $1-2 \%$ of the total dust carbon content (2$4 \times 10^{19}$ carbon atoms, equivalent to a few million dust particles) penetrated the core plasma, raising the core carbon density by a factor of $2-3$. When the OSP was inboard of the dust holder, the dust injection continued at a lower rate. Individual dust particles were observed moving at velocities of $10-100 \mathrm{~m} / \mathrm{s}$, predominantly in the toroidal direction for deuteron flow to the outer divertor target, consistent with the ion drag force. The observed behavior of the dust is in qualitative agreement with modeling by the 3D DustT code.
\end{abstract}


JNM keywords:

PSI-16 keywords: DIII-D, dust

PACS:

*Corresponding author address: 9500 Gilman Dr, Mail code 0417, La Jolla, CA 92093-0417

*Corresponding authore-mail: rudakov@fusion.gat.com

Presenting author address: as above

Presenting author e-mail: as above 


\section{Introduction}

The presence of dust in the fusion plasmas has been long recognized and discussed in literature (see [1-7] and references therein). While generally of no concern in the present day machines, dust may pose serious safety and operational concerns for the next generation of fusion devices such as International Thermonuclear Experimental Reactor (ITER) [4]. Dust generation in the next step devices is expected to increase by a few orders of magnitude due to the increased duty cycle and higher magnitude of particle and power fluxes deposited on the plasma facing components (PFCs) [4]. Dust accumulation inside the vacuum vessel can contribute to tritium inventory rise and cause radiological and explosion hazards [4]. In addition, dust penetrating the core plasma can cause increased impurity concentration and degrade performance [5-7].

Dust particulates found in tokamaks and stellarators range in size between $10 \mathrm{~nm}$ and 100 $\mu \mathrm{m}[4,7]$. The count-based median diameter as well as the diameter of the average mass of the tokamak dust is typically a few microns [4,7]. Dust chemical composition is generally determined by the dominant PFC material. In tokamaks with vacuum chambers lined with carbon tiles ("all-carbon" machines) dust is mostly carbon. Dust in tokamaks is produced by intense plasma material interactions (PMI) during the plasma operations as well as by entry activities during vents. Dust production mechanisms due to PMI include arcing, flaking of codeposited layers, blistering, and brittle destruction [4,7]. Disruptions, "carbon blooms" from leading edges, large ELMs and other transient events result in increased dust production $[4,7]$. Since in a divertor tokamak the strongest PMI usually occur at the divertor targets, dust production rates in the divertor region are expected to be higher than in the rest of the chamber. It was shown by theoretical estimates $[5,6]$ and numerical modeling by the 3D Dust Transport (DustT) code [7] that dust particles formed in the divertor can be accelerated by plasma flows to velocities of $10-100 \mathrm{~m} / \mathrm{s}$ and escape from the divertor region, sometimes 
penetrating the core plasma. Below we provide a direct experimental proof of the long range carbon dust migration in the DIII-D tokamak divertor.

Mobility of dust and its ability to penetrate the core plasma depend strongly on the dust size and chemical composition [5-7]. Validating dust migration models for a known material dust requires knowledge of the dust size and time-resolved trajectory. 2-D imaging techniques [2] can record the dust trajectories and dust velocity can be estimated, but there is usually no way to determine the dust size. Scattering techniques $[3,8]$ can only resolve the size of very small particles, and can not determine velocities. Besides, dust observation events are comparatively rare, on the average often less than one event per plasma discharge. The situation can be meliorated by introducing dust of know composition and size into divertor or scrape-off layer (SOL) and tracing its migration. Experiments with externally introduced dust have been previously performed in JIPPT-IIU tokamak [3], where micron size carbon dust was spread from the top of the vacuum chamber and detected by Thomson scattering. No noticeable effect on the plasma was observed for dust falls of up to at least $10^{6}$ particles (10

$\mu \mathrm{g}$ ) in $20 \mathrm{~ms}$ during discharges. Here we report experiments where much larger amounts of micron size carbon dust were placed in the DIII-D divertor and had a pronounced effect on the plasma discharge.

\section{Experimental arrangement}

DIII-D [9] is a large tokamak ( $R=1.67 \mathrm{~m}, a=0.67 \mathrm{~m}$ ) with all-carbon (graphite) PFCs. DIII-D is equipped with the Divertor Material Evaluation System (DiMES) [10] which allows inserting material samples into the lower divertor floor and exposing them to either a single plasma discharge or a series of reproducible discharges. In the experiments described below, a standard DiMES graphite head was used as a dust sample holder (Fig. 1). A shallow dimple with a smooth depth profile to avoid leading edges, $0.7 \mathrm{~mm}$ deep in the center and $\sim 15 \mathrm{~mm}$ in 
diameter, was made in the plasma-facing (top) surface of the head. Pre-characterized graphite dust consisting of graphite flakes 5-10 $\mu \mathrm{m}$ in diameter, 2-3 $\mu \mathrm{m}$ thick (Fig. 1(a)) was placed in the dimple and the holder was inserted in the lower divertor floor so that its top surface was level with the floor tiles.

Three separate dust exposure experiments have been performed. All exposures were to high power ELMing (featuring Edge Localized Modes) H-mode discharges in lower-singlenull (LSN) magnetic configuration with the strike points swept across the divertor floor. Diagnostic arrangement is illustrated in Figure 2. Three cameras with a view of the dust holder were available during the first two exposures. A tangential view (shown by darker shading in Fig. 2) was split between two CMOS cameras ("tangential TVs") with frame rates of $60 \mathrm{f} / \mathrm{s}$ and changeable filters. One of the cameras had an image intensifier and each frame exposure could be gated down to (1 ms). Another 60 f/s CMOS camera ("DiMES TV”) was viewing the dust holder from the top (view cone showed by lighter shading in Fig. 2). During the last experiment a fast-framing camera with frame rate of $1125 \mathrm{f} / \mathrm{s}$ shared the view with the tangential TVs. One chord of the Multi-chord Divertor Spectroscopy (MDS) system was centered on top of the dust holder (dashed line in Fig. 2). A fiber optic telescope coupled to a photomultiplier with a CIII filter ("filterscope") was focused at a spot at the radial position of DiMES, displaced toroidally by about $40 \mathrm{~cm}$. SPRED spectrometer was monitoring the core CIII radiation. The Charge Exchange Recombination (CER) spectroscopy system was used to measure the core carbon density profiles.

\section{Experimental results}

For the first dust exposure in DIII-D a small amount of loose dust $(\sim 1 \mathrm{mg})$ was placed in the holder, and exposed to a LSN H-mode plasma discharge with strike point sweeps (shot 117294). The outer strike point (OSP) was swept over the dust holder twice, moving first 
inwards then outwards, but no noticeable effect on the discharge parameters was observed, and cameras equipped with $\mathrm{CI}$ and $\mathrm{CII}$ filters did not register any moving dust. The only indication of the dust presence came from MDS that showed step-like increase in both CI line and continuum radiation by about a factor of 3 during the first OSP passage over the holder. CER was not available.

For the second experiment a larger amount of dust, about $30 \mathrm{mg}$, was used. In order to make sure that the dust was not blown away during pump-down and survived until the plasma exposure, a suspension of dust in ethanol was placed in the holder and allowed to dry. Upon drying, it formed a uniform layer clinging to the holder (Fig. 1(b)). The sample was exposed to an LSN H-mode discharge (shot 122428) with the following parameters: toroidal magnetic field, $B_{\mathrm{T}}=2 \mathrm{~T}$, plasma current, $I_{\mathrm{p}}=1.4 \mathrm{MA}$, neutral beam heating power, $P_{\mathrm{NBI}}=5 \mathrm{MW}$, average plasma density, $\bar{n}_{\mathrm{e}}=5 \times 10^{19} \mathrm{~m}^{-3}$. Strike point sweep is illustrated in Fig 2, showing the separatrix positions at $1.5 \mathrm{sec}$ (OSP outboard of DiMES) and $2.0 \mathrm{sec}$ (OSP on DiMES) into the shot. In order to better detect the dust, DiMES TV and the gated tangential TV were equipped with near infrared filters (Kodak Wratten 89B) with less than $1 \%$ transmission below $680 \mathrm{~nm}$. The non-gated ("standard") tangential TV was equipped with a CIII filter.

The first signs of the dust presence came during the startup when DiMES TV registered a bright spot roughly the size of the dust layer on top of the holder. During the current ramp-up the dust holder was kept in the private flux region and no dust injection was occurring. Beginning at $1.72 \mathrm{sec}$ into the discharge the strike points were swept radially inward (Fig. 3(b)). When the OSP reached the dust holder at about $2 \mathrm{sec}$ into the discharge, a massive dust injection occurred. DiMES TV was saturated while the gated tangential TV observed dust trajectories directed towards the plasma core in the poloidal projection plane. Standard tangential TV observed CIII light striated along the magnetic field lines connected to the dust holder. Increase of the continuum radiation from MDS was more than a factor of 50. Both 
SPREAD and divertor filterscope showed increase of CIII light (Fig 3(c,d)). CER observed a strong increase in the core carbon density, peaking at $2.1 \mathrm{sec}$ at more than twice the preinjection level (Fig. 4). Only a minor increase in the average plasma density was observed (Fig. 3(a)).

As the OSP was swept further inwards, the dust injection rate decreased and the carbon light intensity relaxed close to the pre-injection levels. However, dust injection continued at reduced level and DiMES TV registered individual dust trajectories, as illustrated in Fig. 5. No large scale injection was observed on the second, outward directed, OSP pass over the holder. After the second OSP pass dust injection was terminated. The holder was kept inserted for the next plasma discharge with similar plasma parameters and strike point sweeps. Some dust injection was still observed by DiMES TV but the rate was apparently too low to cause any effect on the core plasma. When the holder was removed from DIII-D a small amount of dust was still remaining on the bottom of the dimple.

The third dust injection experiment (shot 123308) was performed in a way similar to the second one, with a similar amount of dust used. The discharge parameters were slightly different: $B_{\mathrm{T}}=2 \mathrm{~T}$, plasma current, $I_{\mathrm{p}}=1.5 \mathrm{MA}$, neutral beam heating power, $P_{\mathrm{NBI}}=8 \mathrm{MW}$, average plasma density, $\bar{n}_{\mathrm{e}}=4 \times 10^{19} \mathrm{~m}^{-3}$. Two major dust injection events were observed: first when the X-point was lowered to induce an L-H transition and landed on top of the dust holder, and second when the OSP was swept across the holder, as in the previous experiments. Both injection events resulted in core carbon density increase by a factor of 2-3. Unlike in previous experiments, both injection events were followed by a notable increase in the average plasma density. While the density increase by a factor of 2.5 after the first injection event was probably mostly due to the L-H transition, the second injection event was most likely responsible for the density increase by about $30 \%$. Dust tracks recorded by the gated tangential TV and fast-framing tangential camera during this event are shown in Figure 6. 


\section{Discussion}

The data collected in the series of experiments described in the previous section allow us to make estimates of the dust velocities and core penetration efficiencies, as well as speculate about the dominant forces defining the dust motion. The low-bound estimate of dust velocities can be done from the standard (60 f/s) camera data by dividing the observed track lengths by the exposure time. The available images yield velocities in the range of $10-100 \mathrm{~m} / \mathrm{s}$. More accurate estimates can be made from the fast framing camera data. In the poloidal projection plane of the camera view, the observed dust velocities were in the $50-200 \mathrm{~m} / \mathrm{s}$ range. Since the toroidal component of the dust velocity is expected to be larger than the poloidal and radial components, this is also a low-bound estimate.

Theoretical estimates [6] and numerical modeling by the DustT code [7] predict the ion drag force to be the dominant force acting on the dust particles in a tokamak divertor and the dust motion being predominantly in the direction of the local ion flow, which in the divertor is mostly toroidal. Images recorded by DiMES TV confirm this. Both theoretical [5,6] and numerical [7] studies predict that dust particles can bounce off the surface inhomogeneities and move towards the plasma core. This was indeed observed by the tangential cameras.

The core penetration efficiency of the dust formed in the divertor is predicted by the DustT modeling to be in the range of a few percent. From the core carbon density increase following the dust injection events, as measured by CER, we can estimate the amount of carbon reaching the core. For the case illustrated in Figure 3, the core carbon density increased by an average of $2 \%$ throughout the shown radial range. For the average plasma density of $5 \times 10^{19} \mathrm{~m}^{-3}$ and plasma volume of $20 \mathrm{~m}^{3}$, this corresponds to $2 \times 10^{19}$ carbon atoms,

or about $4 \times 10^{-4} \mathrm{~g}$. Therefore, during this event about $1.3 \%$ of the total carbon content of the dust (equivalent to a few million dust particles) has reached the plasma core. During the two 
injection events of the third experiment the amount of carbon reaching the core was slightly larger, about $2 \%$ of the total dust content in each event. We should stress that this number is probably not directly comparable to the dust penetration efficiency predicted by the DustT modeling. First, we can not distinguish between the carbon transported to the core in the form of dust (and evaporated inside the core) and that from the dust burned in the boundary plasma and transported into the core by other processes such as diffusion and convection. Second, modeling assumes dust particles having non-zero velocities to begin with (i.e. all the particles are mobile), while in our case there is some finite efficiency of the particle ejection from the holder into the plasma.

\section{Summary and conclusion}

We present direct experimental evidence showing that micron-size carbon dust contained in a tokamak divertor can become highly mobile and reach the core plasma. Whether the dust can be a serious contributor to the core impurity contamination in the present day or future tokamaks remains an open question. The answer would depend on the factors such as dust production and transport rates that are presently poorly known. Our results can serve to validate the models that can be used to predict dust transport in the next step devices such as ITER.

\section{Acknowledgment}

This work was supported by the U.S. Department of Energy under DE-FG02-04ER54758, DE-FC02-04ER54698, W-7405-ENG-48, and DE-AC04-94AL85000. The authors gratefully acknowledge Toyo Tanso Company, Ltd., for supplying the carbon dust. 


\section{References}

[1] R. Behrisch et al., J. Nucl. Mater. 76-77 (1978) 437.

[2] D.H.J. Goodall, J. Nucl. Mater. 111-112 (1982) 11.

[3] K. Narihara et al., Nucl. Fusion 37 (1997) 1177.

[4] G. Federici, et al., Nucl. Fusion 41 (2001)1967.

[5] S.I. Krasheninnikov et al., Phys. Plasmas 11 (2004) 3141.

[6] S.I. Krasheninnikov and T.K. Soboleva, Plasma Phys. Controlled Fusion 47 (2005) A339

[7] A. Yu. Pigarov et al., Phys. Plasmas 12 (2005) 122508.

[8] W.P. West et al., these proceedings.

[9] J.L. Luxon, Nucl. Fusion 42 (2002) 614.

[10] C.P.C. Wong, et al., J. Nucl. Mater. 258-263 (1998) 433. 


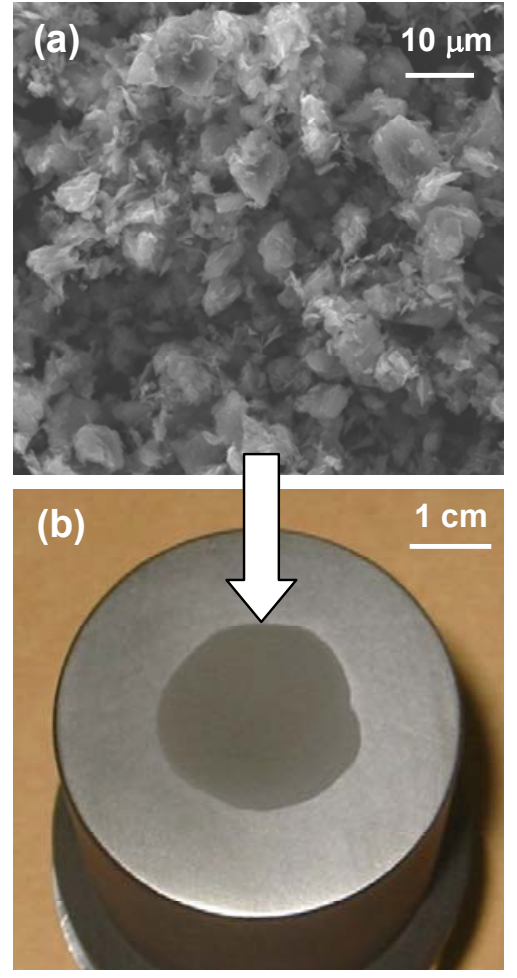

Figure 1: Carbon dust used for experiments in DIII-D (a); dust holder filled with about $30 \mathrm{mg}$ of dust (b).

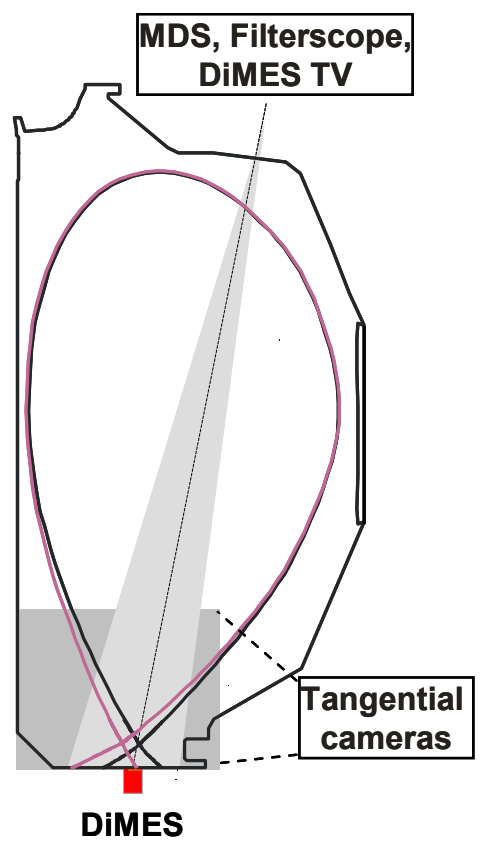

Figure 2: Dust exposure geometry and diagnostic arrangement. 


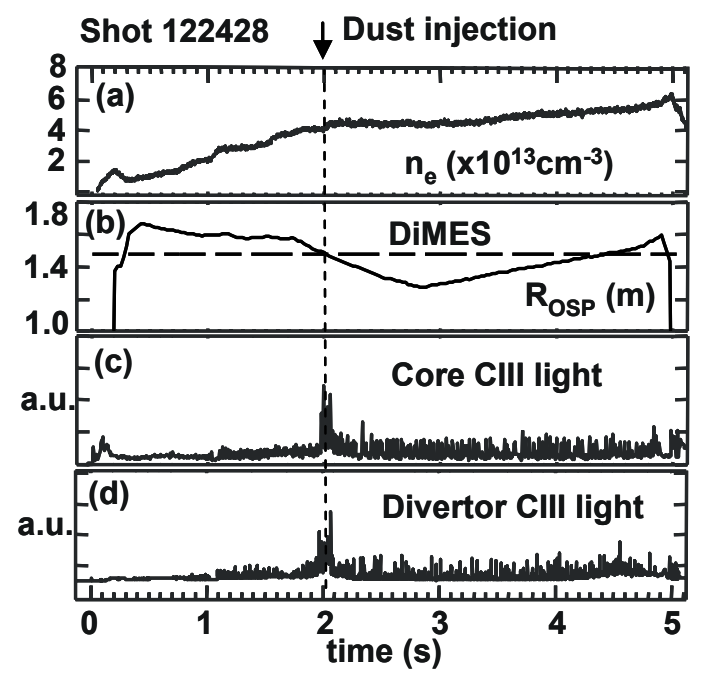

Figure 3: Time traces of the average plasma density (a), radial position of the OSP (b), core (c) and divertor (d) CIII light intensity during a plasma discharge with dust injection. The radial position of DiMES is marked by the horizontal dashed line in (b).

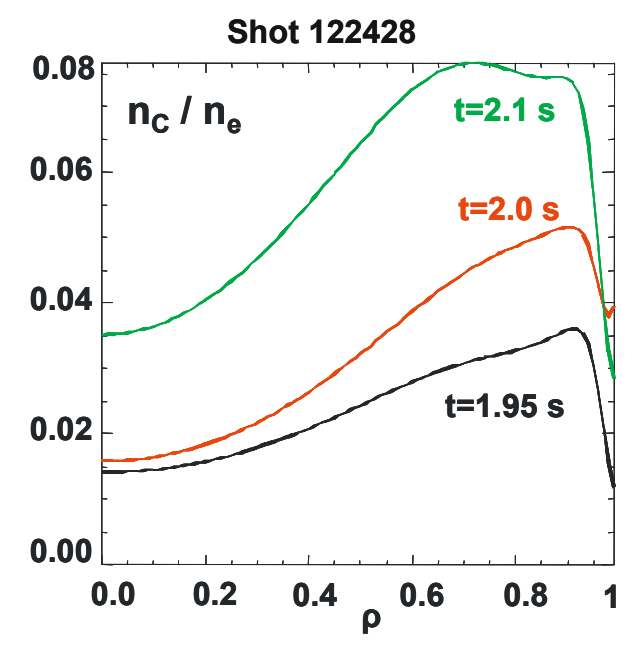

Figure 4: Radial profiles (versus normalized minor radius) of the relative carbon density immediately before, during and after the dust injection. 


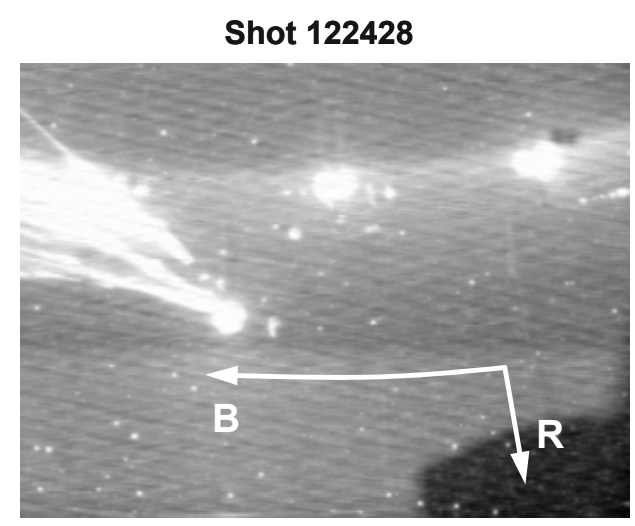

Figure 5: Dust tracks recorded by DiMES TV with near IR filter. Dust holder is the bright spot where the tracks originate.

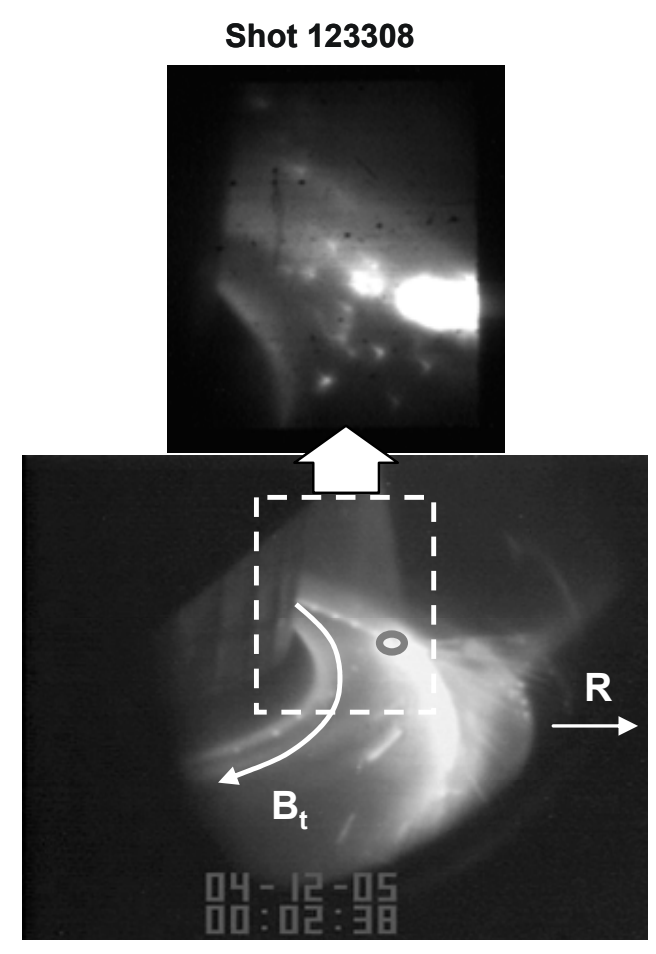

Figure 6: Dust tracks recorded by the gated tangential TV (integration time $3 \mathrm{~ms}$, near IR filter) and fast-framing tangential camera (upper insert, integration time $0.89 \mathrm{~ms}$, no filter). Dust holder position is marked by a circle. 